Cahiers $d u$ MONDE RUSSE

\section{Cahiers du monde russe}

Russie - Empire russe - Union soviétique et États indépendants

$50 / 2-3 \mid 2009$

L'Europe orientale, 1650-1730. Crises, conflits et renouveau

\title{
Defenders of the MotherlandMatthew RENDLE
}

, Oxford, NY : Oxford University Press, 2010, 274 p.

\section{Alexandre Sumpf}

\section{(2) OpenEdition}

\section{Journals}

Édition électronique

URL : https://journals.openedition.org/monderusse/9756

DOI : $10.4000 /$ monderusse. 9756

ISSN : $1777-5388$

Éditeur

Éditions de l'EHESS

Édition imprimée

Date de publication : 15 septembre 2009

ISBN : 978-2-7132-2260-3

ISSN : $1252-6576$

Référence électronique

Alexandre Sumpf, "Defenders of the MotherlandMatthew RENDLE », Cahiers du monde russe [En ligne], 50/2-3 | 2009, mis en ligne le 14 janvier 2013, consulté le 03 septembre 2022. URL : http:// journals.openedition.org/monderusse/9756; DOI : https://doi.org/10.4000/monderusse. 9756

Ce document a été généré automatiquement le 3 septembre 2022

Tous droits réservés 


\title{
Defenders of the MotherlandMatthew RENDLE
}

\author{
, Oxford, NY : Oxford University Press, 2010, 274 p.
}

Alexandre Sumpf

\section{Matthew RENDLE, Defenders of the Motherland, The Tsarist Elite in Revolutionary Russia, Oxford, NY : Oxford University Press, 2010, $274 \mathrm{p}$.}

1 Cet ouvrage, issu de la thèse de l'auteur, se situe à la croisée des récents ouvrages russes sur les "ci-devant ${ }^{16}$ et des monographies anglo-saxonnes sur l'année 1917 dans des régions ${ }^{17}$ ou des groupes sociaux ${ }^{18}$ particuliers. Le plan proposé est à la fois chronologique et thématique, puisqu'il aborde en quatre moments les principales catégories (non exclusives les unes des autres) de l'élite tsariste: nobles, propriétaires terriens et officiers, qui bénéficient chacun d'un chapitre particulier (III, IV, V). Matthew Rendle évalue leur implication spécifique dans les quatre étapes clés du bouleversement radical qu'a alors connu la Russie, liant justement dans un même mouvement la Grande Guerre et les deux révolutions de 1917 (I, II, VII), ainsi que la tentative de putsch du général Kornilov (VI). L'historien fait la part des choses entre accusations répétées de "contre-révolution», lancées par les révolutionnaires, discours publics (presse, congrès), pensées intimes (carnets, lettres) et responsabilités réelles dans un certain nombre d'événements révélateurs.

2 Rendle débute son étude au lendemain de la première révolution russe, arguant que « les élites ont adopté les méthodes de la politique de l'après 1905 (organisations, meetings, publications, etc.) plus vite que les nouvelles idéologies»(p. 17). Tout au long de l'ouvrage, on voit en effet les nobles se constituer en associations et mener des enquêtes par questionnaire, les propriétaires terriens (et immobiliers) réunir des congrès avec des délégués venus de tout le pays et adresser des pétitions au gouvernement, les officiers jusqu'alors tenus au droit de réserve s'organiser en un large spectre politique de mouvements après Février. Tous jouent le jeu, pas seulement celui de la démocratisation, 
comme l'assure l'auteur, mais surtout de l'autonomisation de la société civile et de la constitution de forces sociales, dont l'origine remonte aux premières associations et sociétés savantes du milieu du XIX ${ }^{\mathrm{e}}$ siècle.

La Première Guerre mondiale achève de désolidariser les trois composantes de l'élite de la famille impériale, et du tsar en particulier. Si Rendle ne revient pas sur le cas Rasputin, il met en évidence le fossé qui se creuse au nom de l'intérêt national, interprété de manière différente par les progressistes peu attachés à la monarchie et le "Bloc noir " qui cherche encore une issue impériale à la crise de gouvernance. Sans avoir participé directement aux événements de Février, les élites soutiennent d'emblée un Gouvernement Provisoire dont la compétence et la responsabilité suscitent l'espoir. Une fêlure apparaît toutefois entre officiers de différents grades et origines sociales (les pertes de guerre et les actes de bravoure ont accéléré les promotions, notamment parmi les $80 \%$ d'officiers qui étaient de simples enseignes), lourde de conséquences à la fois dans l'affaire Kornilov et le succès du coup d'État bolchevik.

4 La révolution sociale des campagnes met à rude épreuve le pacte nouvellement scellé autour de la défense de la patrie et de la propriété privée. Les nobles organisés en groupe d'influence doivent accepter la remise en cause de leur statut de garants de l'ordre social (les maréchaux de la noblesse) et le fait qu'ils ne représentent plus qu'un groupe social parmi d'autres. Or, tandis que la Douma devient le foyer de la résistance à la révolution, des nobles socialistes de longue date ou des officiers convaincus soutiennent des mouvements qui menacent leur caste au quotidien (les domestiques se syndiquent, les nobles ne peuvent plus vivre de leurs rentes et les licencient) et à plus long terme. On relève l'incapacité de la noblesse, en dépit de tentatives locales, à participer vraiment aux élections - faute d'unité bien sûr, mais surtout à cause de l'inertie de la majorité, incapable de réagir avec l'intensité et la rapidité voulues à l'offensive de l'égalitarisme.

5 Les élites, en particulier les propriétaires, font le choix de l'approche stolypinienne multiplier les petits propriétaires plutôt que redistribuer la terre à parts égales à l'ensemble de la paysannerie - sans doute trop tard, d'autant qu'ils se voient exclus des comités agraires. Ils lancent alors une offensive sur le plan légal, ce qui renforce la cohésion du groupe des propriétaires, mais ne donne pas les résultats escomptés, tant le gouvernement se trouve dans l'incapacité de se faire respecter en province. Reconnaître cette faiblesse, c'est envisager une solution de remplacement qui passe par l'appel aux officiers.

Or ceux-ci se trouvent divisés entre plusieurs associations et affiliations politiques, sur fond de passivité et de désillusion grandissante. Ainsi, l'Union des officiers républicains mélange socialistes radicaux et modérés qui soutiennent le Gouvernement Provisoire jusqu'au 26 avril, date à laquelle elle se renomme Union des socialistes de l'armée du peuple. Le Soviet des députés officiers continue, lui, à soutenir la Douma, le ministère de la Guerre et le travail de la Commission Polivanov sur les rapports entre officiers et soldats, mais échoue à se structurer en mouvement national. Deux congrès concurrents d'officiers organisés en même temps (mai 1917) à Petrograd et à l'état-major entérinent la fracture entre ceux qui militent pour une professionnalisation de l'armée (et pour les droits politiques des officiers), et ceux qui entendent maintenir son rôle dans le strict cadre de la guerre et de l'obéissance au pouvoir civil. Outre le conflit générationnel, les positions des officiers expriment le fossé séparant servants d'active au front et gardiens de garnison. Deux associations tentent de transcender la séparation entre officiers et soldats du rang: la Ligue militaire, au discours plus patriotique et conservateur, milite 
avant tout pour la discipline militaire ; l'Union des cavaliers de Saint-Georges appelle à aller au bout du sacrifice consenti. En dépit des publications et des congrès, du lobby exercé, de la volonté de jouer le jeu démocratique, l'ensemble de ces organisations peine à se structurer au plan national et à véritablement représenter le corps des officiers.

7 L'échec d'une solution civile - fondée sur la classe, la nationalité, le parti ou même le type de métier militaire - pour régler ces conflits, favorise l'apparition d'une solution militaire qui, par son insuccès, casse définitivement la dynamique unificatrice. Le putsch tenté par le général Kornilov n'obtient pas le soutien massif des élites, y compris chez les officiers. L'option de la dictature ne l'emporte ni sur celle d'un gouvernement fort d'union nationale, au-dessus des classes et des partis, ni sur celle du retour à la monarchie - dépourvue de candidat probant. Le rare soutien actif à Kornilov tient plus à son autorité personnelle qu'à la conviction que le putsch doit et va réussir. La plupart des membres de l'élite, en faveur de la suppression du Soviet et critiques quant à l'impuissance du gouvernement, se révèlent trop effrayés pour agir. En dépit d'une rapide prise de distance avec Kornilov, l'affaire nuit un peu plus à l'image des officiers et des propriétaires. Ces derniers placent désormais tous leurs espoirs dans la Constituante, allant jusqu'à solliciter les paysans "séparateurs" (otrubniki), qui sont aussi les principales victimes du « mouvement agraire » du printemps et de l'automne 1917.

8 La rhétorique des bolcheviks et la violence exercée envers les «ci-devant » (byvšie), la privation de droits civiques (lišency), le décret sur la terre et la guerre civile naissante achèvent de marginaliser les trois composantes de l'élite, qui ont pourtant globalement accepté l'abdication de Nicolas II et la faible probabilité d'un régime autocratique. En ce sens, conclut l'auteur, les élites n'ont pas été aussi conservatrices qu'on l'a dit ; en tout cas, pas réactionnaires, ni, dans leur majorité, «contre-révolutionnaires». Dans l'ensemble, elles ont manifesté de larges hésitations, leurs concessions à la démocratie ont toujours été faites avec retard par rapport au mouvement révolutionnaire et elles ne sont jamais parvenues à représenter plus qu'elles-mêmes, c'est-à-dire une infime minorité.

9 L'ouvrage de Rendle, qui synthétise plusieurs décennies d'historiographie, a le mérite d'attirer l'attention sur les élites, mais pèche à plusieurs égards. Tout d'abord, si la religion a droit de cité ici et là, le clergé ne fait pas l'objet d'une étude aussi poussée que les nobles ou les officiers. Or, l'Église orthodoxe jouait un rôle non négligeable dans la stabilité du pouvoir autocratique mis à bas en Février et, à chaque échelle territoriale et socioéconomique, le clergé se rangeait dans la couche supérieure de la population. Ensuite, l'appel à des exemples provinciaux ne permet pas l'économie d'une réflexion capitale sur les différences « nationales » et surtout régionales - ne serait-ce qu'entre les deux capitales. Qu'est-ce qui explique, par exemple, que la plupart des congrès se réunissent à Moscou, alors que Douma, Soviet et Gouvernement siègent à Petrograd? Enfin, les sources convoquées, en particulier provenant de fonds d'archives, ne sont ni assez diverses ni assez systématiquement étudiées, comme la voix des membres moins connus de l'élite : une analyse de la correspondance d'un des nombreux organes de presse ou des journaux intimes d'une population lettrée à $100 \%$ aurait sans doute contribué à affiner encore les aspects de son originalité dans la société russe révolutionnée. 


\section{NOTES}

16. Sof'ja A. Čujkina, Dvorjanskaja pamjat': "Byvšie» v sovetskom gorode (Leningrad, 1920-30-ye gody) [La mémoire de la noblesse: "Les ci-devant» dans la ville soviétique (Leningrad, 1920-1930)], SPb. : Evropejskij universitet v Sankt-Peterburge, 2006.

17. Donald Raleigh, Experiencing Russia's Civil War: Politics, Society and Revolutionnary Culture in Saratov, Princeton: Princeton University Press, 2002 ; Sarah Badcock, Politics and the People in Revolutionary Russia: A Provincial History, Cambridge: Cambridge University Press, 2007.

18. Aaron B. Retish, Russia's Peasants in Revolution and Civil War: Citizenship, Identity, and the Creation of the Soviet State, 1914-1922, Cambridge : Cambridge University Press, 2008. 